\title{
Bowel obstruction caused by an internal hernia that developed after laparoscopic subtotal colectomy: a case report
}

Takefumi Yoshida*, Tetsushi Kinugasa, Yousuke Oka, Tomoaki Mizobe, Hiroto Ishikawa, Naoki Mori, Taro Isobe, Eri Katayama and Yoshito Akagi

\begin{abstract}
Introduction: Laparoscopic surgery is a minimally invasive approach with good treatment outcomes and is currently the standard surgery for colorectal cancer in Japan. Mesenteric closure is considered unnecessary in laparoscopic colorectal surgery because it can damage the bowel and blood vessels. However, an internal hernia may develop if the mesentery is not repaired.

Case presentation: We report a case of internal hernia in a 61-year-old male of Japanese ethnicity. The patient had advanced sigmoid colon cancer, early-stage transverse colon cancer, and multiple adenomatous polyposis, and underwent laparoscopically-assisted subtotal colectomy. Bowel obstruction developed six days postoperatively and did not improve with conservative treatment. Abdominal computed tomography detected an internal hernia, prompting emergency surgery in which the ileum protruding into the mesenteric defect and an anastomotic stricture were detected. Reanastomosis, mesentery closure, and ileostomy were performed after hernia repair.

Conclusion: In this case, open surgery was necessary due to bowel obstruction after laparoscopic colectomy. This outcome indicated that mesenteric closure should have been performed. Thus, the benefits of mesenteric closure require assessment in future cases.
\end{abstract}

Keywords: Laparoscopic surgery, Subtotal colectomy, Bowel strangulation, Internal hernia, Mesenteric closure

\section{Introduction}

Laparoscopic surgery for colon cancer yields treatment outcomes equivalent to those in open surgery, and thus this approach has been rapidly adopted [1]. Laparoscopic surgery also benefits patients because it leaves a smaller scar than open surgery and is minimally invasive [2]. However, some negative effects can arise as a result of conditions unique to laparoscopic surgery, including rising intraperitoneal pressure due to pneumoperitoneum and steep head-up or head-down positions, and there have been reports of development of internal hernias if the mesentery is not repaired [3-8]. The mesenteric defect is typically closed after open surgery, but this is generally not considered to be necessary after laparoscopic surgery [6]. Here, we report a case of bowel

\footnotetext{
* Correspondence: yoshida_takefumi@med.kurume-u.ac.jp Department of Surgery, Kurume University School of Medicine, 67 Asahi-machi, Kurume-shi, Fukuoka-ken 810-0023, Japan
}

obstruction caused by an internal hernia that developed after laparoscopic subtotal colectomy without mesenteric repair.

\section{Case presentation}

The patient was a 61-year-old male of Japanese ethnicity. He was $167 \mathrm{~cm}$ tall, weighed $42 \mathrm{~kg}$, and had a body mass index (BMI) of $15 \mathrm{~kg} / \mathrm{m}^{2}$. He was admitted to our hospital to undergo surgery for multiple colorectal tumors. Colonoscopy revealed sigmoid colon cancer, an early cancer in the transverse colon, and several adenomas across the length of the entire colon (Figure 1). Laparoscopic subtotal colectomy was performed under general and epidural anesthesia, after informed consent was obtained from the patient. The inferior mesenteric artery and vein were resected distal the left colic artery branch. The right and left side of the colon and each mesentery and the hepatic and splenic flexure were mobilized after 


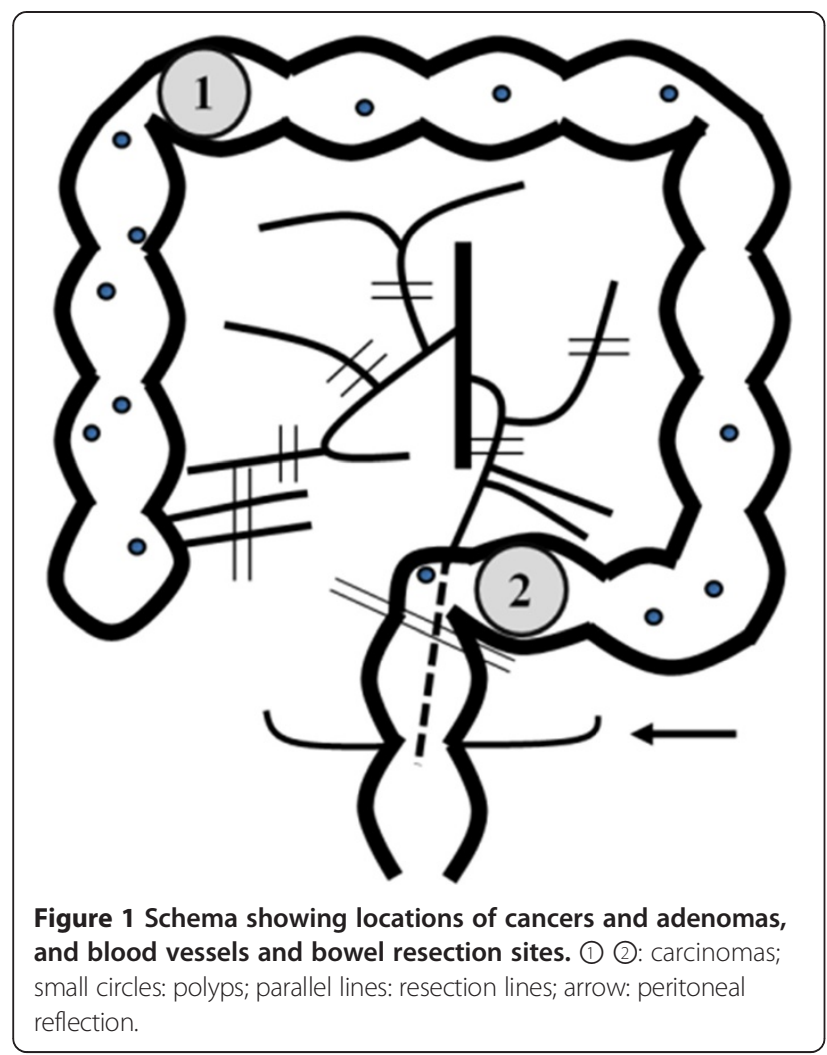

the area between the transverse colon and the greater omentum was resected. During detachment of the splenic flexure, all layers of the transverse colon were damaged and the intestinal contents flowed out. The ileocolic artery and vein, and the right colic and middle colic vessels were resected at each trunk. The rectum was detached up to the peritoneal reflection. Ten centimeters of the colon to the anal side of the sigmoid colon cancer was transected using a linear stapler (Echelon Flex $^{\text {tm }}$ Powered Endopath Stapler 60; Ethicon, USA). After the colon was pulled out from the umbilical region and the ileum end was transected, the colon was excised. Side-to-end ileorectal anastomosis was performed using a circular stapler (Proximate* ILS CDH 29; Ethicon, USA) (Figure 2). Penrose drains were inserted below the left crus of the diaphragm and at the posterior surface of the anastomosis after the peritoneal cavity was washed with 3,000 cc of warm physiological saline. The small bowel was returned to its original position, antiadhesive material was placed inside the cavity, and the abdomen was closed. The duration of surgery was $8 \mathrm{~h}$ and $22 \mathrm{~min}$, with $200 \mathrm{cc}$ of blood loss.

The final diagnosis was T2NOMO stage I moderately differentiated adenocarcinoma for the sigmoid colon cancer, stage 0 (TisNOM0) well differentiated adenocarcinoma for the transverse colon cancer, and tubular adenomas (10 low grade adenomas, 3 high grade adenomas) for other polyps. Bowel obstruction developed on the sixth

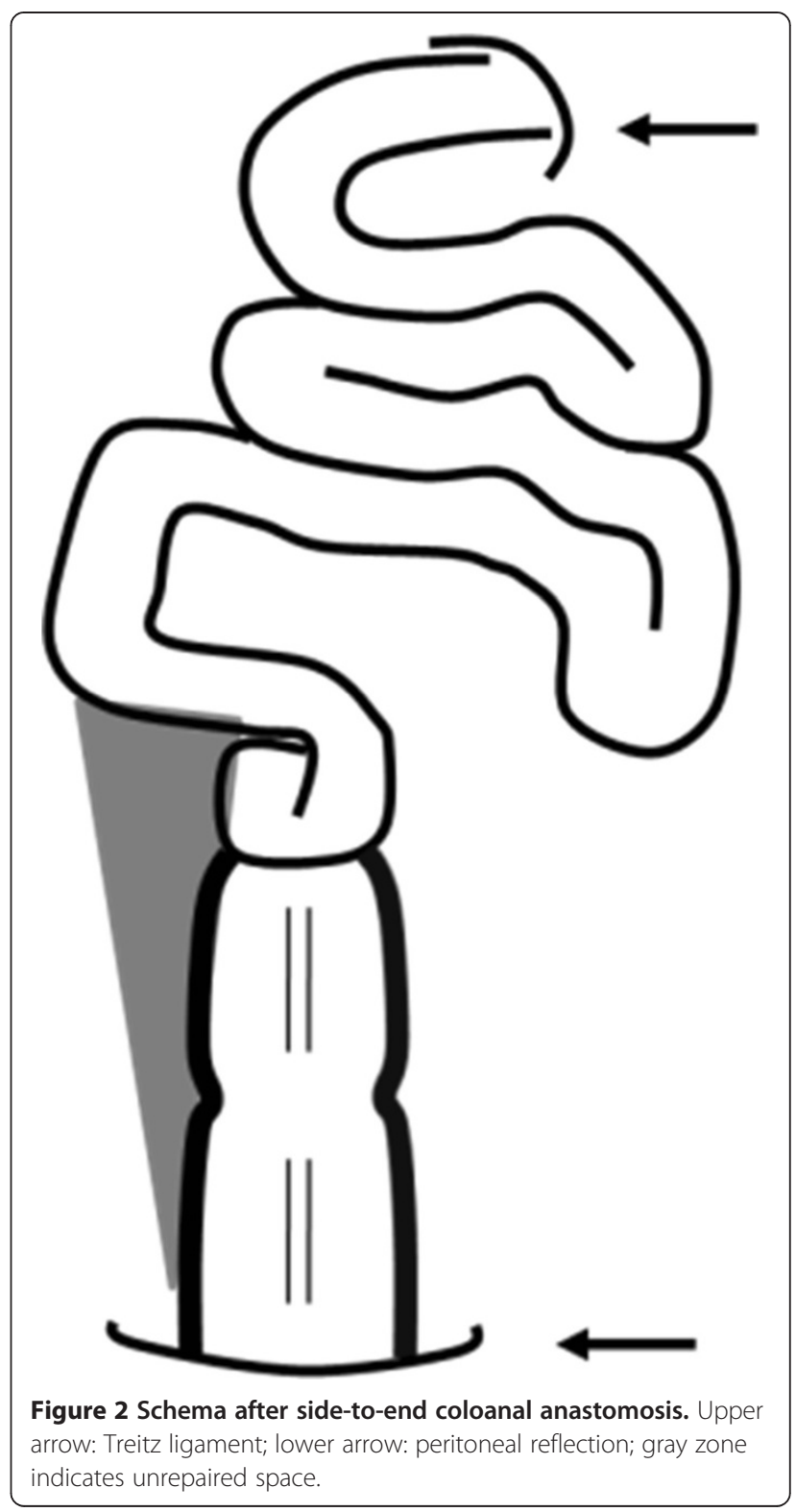

day after surgery (Figure 3) and did not improve, even after an ileus tube was inserted and conservative treatment was performed. He had fever and abdominal distension. Blood tests (Table 1) revealed an elevated inflammatory response, hypoalbuminemia, impaired liver function, and coagulation system abnormalities.

Emergency surgery was performed because bowel obstruction caused by internal hernia was suspected based on abdominal computed tomography (CT) findings (Figure 4). The upper part of the small bowel was dilated because the ileum had protruded into the mesenteric defect, leading to an internal hernia. The tip of the ileus tube had stopped at the oral side of the incarcerated herniated intestine (Figures 5 and 6). The area around the anastomosis had hardened and was determined to be an anastomotic 


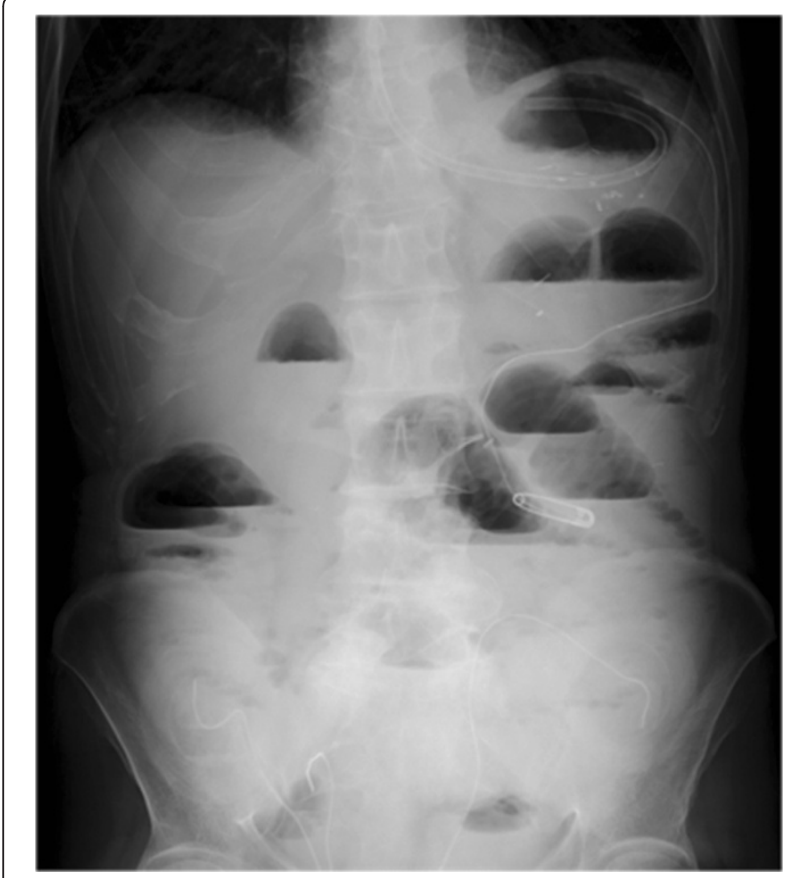

Figure 3 Plain standing abdominal X-ray. Niveau formation in the small intestine.

stricture. The incarcerated herniated bowel was $30 \mathrm{~cm}$ long, whereas the ileum to the oral side of the ileorectal anastomosis to the anal side was $40 \mathrm{~cm}$ long. Re-anastomosis was performed after hernia repair because the situation was treated as a stenosis, even though circulation to the bowel of the ileorectal anastomosis was not interrupted. The mesenteric defect was closed using 3-0 Vicryl, and a Penrose drain was inserted into the posterior of the anastomosis. A loop ileostomy was them constructed. The patient began eating on the third day after emergency surgery and recovered with no major problems. He was discharged 28 days after surgery.

\section{Discussion}

In recent years, there has been an increase in the number of laparoscopic surgeries for colorectal cancer in Japan, as shown by the results of a survey conducted by the Japan Society for Endoscopic Surgery [9]. Laparoscopic surgery has been proven to be comparable with open surgery and is becoming the standard method for colorectal cancer surgery [1,2]. Aggregate data in Japan indicates that 82,291 patients with benign or malignant conditions of the small or large bowel underwent laparoscopic surgery from 2008 to 2011 , of which $4,912(6 \%)$ experienced a procedural accident, including 1,172 cases (1\%) of bowel obstruction [9]. Bowel obstruction caused by an internal hernia after laparoscopic surgery has been reported in Roux-en-Y reconstruction [10-12], Nissen fundoplication [13], and various types of laparoscopic urogenital surgeries [14].
Table 1 Blood test findings before emergency surgery at 6 days after subtotal colectomy

\begin{tabular}{|c|c|}
\hline Item & Value \\
\hline White blood cells WBC $(/ \mu \mathrm{L})$ & 13800 \\
\hline Red blood cells RBC $\left(10^{4} / \mu \mathrm{L}\right)$ & 333 \\
\hline Hemoglobin $(\mathrm{Hb})(\mathrm{g} / \mathrm{dL})$ & 11.4 \\
\hline Hematocrit (Ht) (\%) & 31.4 \\
\hline Platelets (Plt) $\left(10^{4} / \mu \mathrm{L}\right)$ & 58.2 \\
\hline Albumin (Alb) (g/dL) & 3.03 \\
\hline Total bilirubin (T.Bil) (mg/dL) & 0.44 \\
\hline Aspartate aminotransferase (AST) (U/L) & 141 \\
\hline Alanine aminotransferase (ALT) (U/L) & 140 \\
\hline Lactic acid dehydrogenase (LD) (U/L) & 215 \\
\hline Alkaline phosphatase (ALP) (U/L) & 386 \\
\hline Y-Glutamyltranspeptidase ( $(\gamma-G T P)(U / L)$ & 81 \\
\hline Blood urea nitrogen $(\mathrm{BUN})(\mathrm{mg} / \mathrm{dL})$ & 10 \\
\hline Creatinine (Cre) (mg/dL) & 0.45 \\
\hline Sodium (Na) (mmol/L) & 126 \\
\hline Chloride $(\mathrm{Cl})(\mathrm{mmol} / \mathrm{L})$ & 87 \\
\hline Potassium (K) (mmol/L) & 5.2 \\
\hline Amylase (Amy) (U/L) & 79 \\
\hline C-reactive protein test (CRP) (mg/dL) & 8.26 \\
\hline Prothrombin time (PT) (\%) & 71 \\
\hline Activated partial thromboplastin time (APTT) (sec) & 42.8 \\
\hline Fibrin and fibrinogen degradation product (FDP) $(\mu \mathrm{g} / \mathrm{mL})$ & 21.2 \\
\hline
\end{tabular}

Data shown in bold are elevated from normal levels.

There are also several reports of internal hernia after laparoscopic colorectal cancer surgery [3-8]. Among these, Cabot et al. [6] observed 4 cases of internal hernia in 530 laparoscopic right colon surgeries $(0.8 \%)$ and Trabaldo et al. [7] observed 5 cases in 436 laparoscopic left colon surgeries (1.14\%). Only 2 deaths were reported in these studies $[6,7]$, but the individual risk is uncertain. A narrow defect hole $(2-5 \mathrm{~cm})$ caused by incomplete closure may increase the risk of a symptomatic internal hernia [8]. Internal hernia in our patient might have developed due to the long duration of surgery; or may have been caused by protrusion of the bowel into the mesenteric defect due to the higher intraperitoneal pressure during postoperative bowel paresis or intraperitoneal infection. Given the low incidence of internal hernias, mesenteric closure may not be necessary for every patient. However, closure should be considered in bowel damage during surgery if postoperative bowel paresis is likely or if the patient has a thin physique, as for our patient. Masubuchi et al. [3] proposed that the mesenteric defect should be closed in thin patients because they are at risk for developing an internal hernia. 

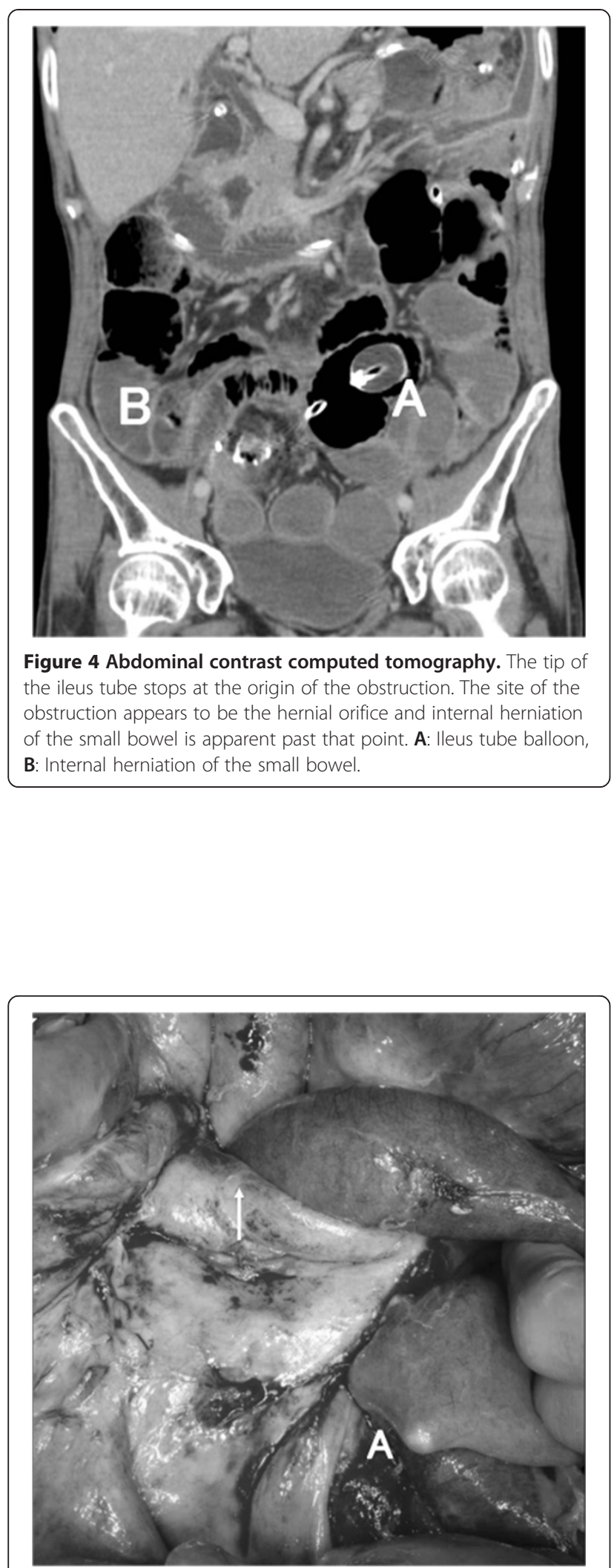

Figure 5 The small bowel protruded into the opening of the hernia (arrow), which was formed by the mesenteric defect. A: Tip of the ileus tube. The top of the image is the cranial side.

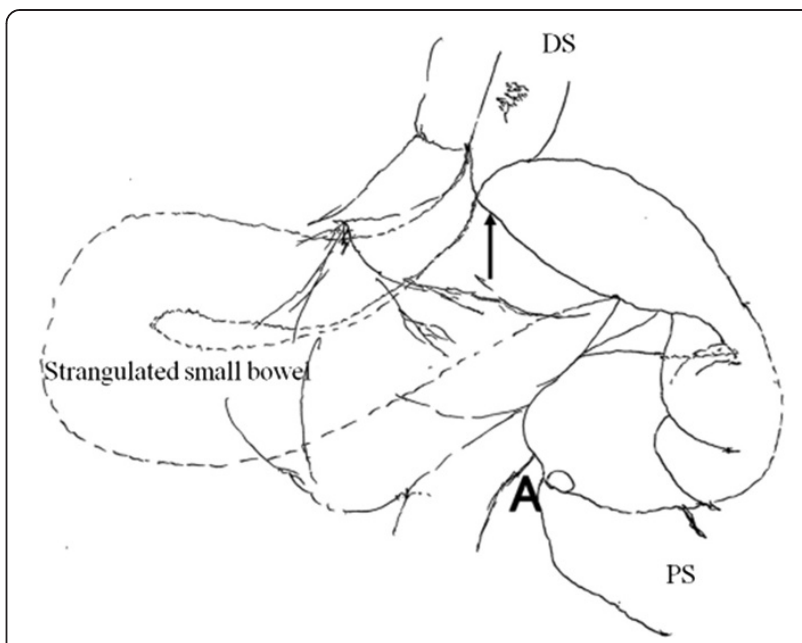

Figure 6 Schema of Figure 5. Dotted line shows the protruding section of the small bowel. Arrow: hernial orifice; $\mathbf{A}$ : ileus tube; DS: distal side; PS: proximal side.

The current case shows that both frontal and transverse CT images are useful for diagnosis of internal hernias. Trabaldo et al. [7] also used CT scans for diagnosis. In our case, bowel obstruction arose from an internal hernia caused by the absence of repair of the mesentery after laparoscopic subtotal colectomy. Diagnosis with CT permitted subsequent performance of emergency surgery.

\section{Conclusion}

We have reported a case of internal hernia that developed after laparoscopic surgery for colon cancer. This case suggests that the benefits of mesenteric closure after laparoscopic colorectal surgery should be examined further in patients with risk factors for development of internal hernia. In such patients, postoperative abdominal computed tomography may be useful for detection of a potential hernia.

\section{Consent}

Written informed consent was obtained from the patient for publication of this case report and accompanying images. A copy of the written consent is available for review by the Editor-in-Chief of this journal.

\section{Abbreviations}

BMI: Body mass index; CT: Computed tomography.

\section{Competing interest}

The authors declare that they have no competing interests.

\section{Authors' contributions}

$T Y$ is a surgery of this case, drafted the manuscript. KY carried out advice. YO collected the reference paper. TM confirmed the pathological findings. HI was assistant of emergency surgery. NM carried out translation. TI subjected to peer review. EK carried out before and after surgery management. AY is the planner of this paper, he carried out the command to create the paper. All authors read and approved the final manuscript. 


\section{Acknowledgments}

The authors would like to thank Emeritus Professor Kazuo Shirouzu for his

continued support and constant encouragement.

Received: 21 October 2014 Accepted: 8 December 2014

Published: 29 December 2014

\section{References}

1. The Clinical Outcomes of Surgical Therapy Study Group: A comparison of laparoscopically assisted and open colectomy for colon cancer. N Engl J Med 2004, 350:2050-2059.

2. Lacy AM, Garcia-Valdecasas LC, Delqado S, Castells A, Taura P, Pique JM, Visa J: Laparoscopy-assisted colectomy versus open colectomy for treatment of non-metastatic colon cancer: a randomized trial. Lancet 2002, 359:2224-2229.

3. Masubuchi S, Okuda J, Tanaka K, Kondo K, Asai K, Kayano H, Yamamoto M, Uchiyama K: An internal hernia projecting through a mesenteric defect following laparoscopic-assisted partial resection of the transverse colon to the lesser omental cleft: report of a case. Surg Today 2013, 43:814-817.

4. Blanc P, Delacoste F, Atger J: A rare cause of intestinal obstruction after laparoscopic colectomy. Ann Chir 2003, 128:617-621.

5. Elio A, Veronese E, Frigo F, Residori C, Salvato S, Orcalli F: lleal volvulus on internal hernia following left laparoscopic-assisted hemicolectomy. Surg Laparosc Endosc 1998, 8:477-478.

6. Cabot JC, Lee SA, Yoo J, Nasar A, Whelan RL, Feingold DL: Long-term consequences of not closing the mesenteric defect after laparoscopic right colectomy. Dis Colon Rectum 2010, 53:289-292.

7. Sereno Trabaldo S, Anvari M, Leroy J, Marescaux J: Prevalence of internal hernias after laparoscopic colonic surgery. J Gastrointest Surg 2009, 13:1107-1110.

8. Hosono $\mathrm{S}$, Ohtani $\mathrm{H}$, Arimoto $\mathrm{Y}$, Kanamiya $\mathrm{Y}$ : Internal hernia with strangulation through a mesenteric defect after laparoscopy-assisted transverse colectomy: report of a case. Surg Today 2007, 37:330-341.

9. Japan Society for Endoscopic Surgery: Aggregate results of the 11th Nationwide Survey of Endoscopic Surgery in Japan. J Jpn Soc Endosc Surg 2012, 17:571-611.

10. Steele KE, Prokopwicz GP, Magnuson T, Lidor A, Schweitzer M: Laparoscopic antecolic Roux-en-Y gastric bypass with closure of internal defects leads to fewer internal hernias than the retrocolic approach. Surg Endosc 2008, 22:2056-2061.

11. Coleman MH, Awad ZT, Pomp A, Gagner M: Laparoscopic closure of the Petersen mesenteric defect. Obes Surg 2006, 16:770-772.

12. Row D, Maddineni S, Maffucci L, Rangraj M: Late sigmoid colon internal herniation into the jejuno-jejunostomy mesenteric defect after laparoscopic Roux-en-Y gastric bypass. Obes Surg 2006, 16:208-210.

13. Malas MB, Katkhouda N: Internal hernia as a complication of laparoscopic Nissen fundoplication. Surg Laparosc Endosc Percutan Tech 2002, 12:115-116.

14. Wasserman SA: Incarcerated hernia after laparoscopically assisted vaginal hysterectomy. J Am Assoc Gynecol Laparosc 1994, 1:415-416.

doi:10.1186/1752-1947-8-470

Cite this article as: Yoshida et al:: Bowel obstruction caused by an internal hernia that developed after laparoscopic subtotal colectomy: a case report. Journal of Medical Case Reports 2014 8:470.

\section{Submit your next manuscript to BioMed Central and take full advantage of:}

- Convenient online submission

- Thorough peer review

- No space constraints or color figure charges

- Immediate publication on acceptance

- Inclusion in PubMed, CAS, Scopus and Google Scholar

- Research which is freely available for redistribution 